\title{
Risk Factors for Delirium after Spine Surgery: An Age-Matched Analysis
}

\author{
Tadao Morino ${ }^{1}$, Masayuki Hino ${ }^{1}$, Shintaro Yamaoka ${ }^{1}$, Hiroshi Misaki ${ }^{1}$, \\ Tadanori Ogata ${ }^{1}$, Hiroshi Imai ${ }^{2}$, Hiromasa Miura ${ }^{2}$ \\ ${ }^{I}$ Spine Center, Ehime University School of Medicine, Ehime, Japan \\ ${ }^{2}$ Department of Orthopedic Surgery, Ehime University School of Medicine, Ehime, Japan
}

\begin{abstract}
Study Design: A retrospective cohort study.
Purpose: To investigate the risk factors for postoperative delirium after spine surgery, excluding older age, which has already been established as a strong risk factor.

Overview of Literature: More than 30 risk factors have been reported for delirium after spine surgery, making it challenging to identify which factors should be prioritized. We hypothesized that risk factors could not be prioritized to date because the factor of older age is very strong and influenced other factors. To eliminate the influence of older age, we performed an age-matched group comparison analysis for the investigation of other risk factors.

Methods: This study involved 532 patients who underwent spine surgery. Two patients of the same age without delirium (delirium negative group) were matched to each patient with delirium (delirium positive group). Differences in suspected risk factors for postoperative delirium between the two groups identified from previous reports were analyzed using univariate analysis. Multivariate analysis was performed for factors that showed a significant difference between the two groups in the univariate analysis.

Results: Fifty-nine (11.1\%) of 532 patients developed postoperative delirium after spine surgery. Large amounts of intraoperative bleeding, low preoperative concentration of serum $\mathrm{Na}$, high postoperative (day after surgery) serum level of C-reactive protein, low hematocrit level, low concentration of albumin, and high body temperature were detected as significant risk factors in the univariate analysis. Large amounts of intraoperative bleeding remained a risk factor for postoperative delirium in the multivariate analysis.

Conclusions: We should pay attention to and take precautions against the occurrence of postoperative delirium after spine surgery in patients of older age or those who experience severe intraoperative bleeding.
\end{abstract}

Keywords: Spine; Delirium; Risk factors; Multivariate analysis; Hemorrhage

\section{Introduction}

Delirium is characterized by disturbances in attention and awareness associated with impairment in at least one cognitive function, including memory, orientation, language, visuospatial ability, or perception. These disturbances develop suddenly and fluctuate throughout the day [1]. Surgical stress associated with general anesthesia may cause a type of delirium called postoperative delirium, a clinical symptom characterized by acute attentional deficit experi-

Received Nov 27, 2017; Revised Dec 12, 2017; Accepted Dec 17, 2017

Corresponding author: Tadao Morino

Spine Center, Ehime University, Shitsukawa, Toon City, Ehime, 791-0295, Japan

Tel: +81-89-960-5343, Fax: +81-89-960-5346, E-mail: morino@m.ehime-u.ac.jp 
enced postoperatively. In general, postoperative delirium occurs after postoperative day 1 [2].

Identifying the possible risk factors for postoperative delirium may be helpful for its prevention. A large number of risk factors for postoperative delirium have been reported in connection with various types of surgery, including cardiovascular interventions, surgery for hip fracture, and total hip or knee arthroplasty [3-5]; therefore, it is unclear which factors should be considered when predicting the occurrence of this condition. Shi et al. [6] performed a meta-analysis of risk factors for postoperative delirium after spine surgery. Based on a univariate analysis of factors reported in different studies, they concluded that the most significant risk factors were older age ( $>60$ years), female sex, polypharmacy, preoperative lower hematocrit (Hct) and albumin (Alb) levels, increased operative time and blood loss, postoperative hyponatremia, postoperative low hemoglobin $(\mathrm{Hb})$ level, postoperative low Hct level, and fever. Among these, older age has been identified as a risk factor in all previous studies. Therefore, we performed an age-matched retrospective cohort study to detect risk factors for delirium after spine surgery, excluding older age.

\section{Materials and Methods}

\section{Patients}

This study involved 532 patients who underwent spine surgery at Ehime University Hospital from 2012 to 2014. There were 283 males and 249 females, with an average age of 64.2 years (range, 10-89 years). None of the patients had delirium or a history of delirium preoperatively. None of the patients had a history of psychiatric diseases requiring the use of psychotropic drugs. All operations were performed by one of the four senior spine surgeons having $>10$ years of clinical experience. All operations were performed under general anesthesia with propofol and isoflurane, enflurane complex with fentanyl, and rocuronium bromide or vecuronium bromide, and all the patients were supplied with oxygen (3-5 L/min) through a mask for at least 24 postoperative hours. The study was approved by the institutional review board at Ehime University (IRB approval no., 1801010).

\section{Evaluation of delirium}

The presence of delirium was determined based on the di- agnostic and statistical manual of mental disorders (DSMIV) criteria [7], which are the gold standard for psychiatric evaluation [8]. Nurses were trained for the detection of symptoms, including hyperactivity, hypoactivity, disarray, hallucination, and delusion, in patients according to the DSM-IV criteria. Patients both with and without symptoms of delirium were observed during regular postoperative care from day 0 to 7 . When a patient was suspected with symptoms of delirium, the nurse called two doctors to discuss whether the patient actually had delirium. Patients were finally diagnosed as having delirium when both the doctors confirmed the diagnosis. The presence of delirium and its symptoms were recorded in the medical records after the decision.

\section{Evaluation involving all the patients}

The clinical records of all 532 patients were retrospectively reviewed, and patients were divided into two groups: delirium positive and negative groups. The overall and age group-related incidences of delirium were calculated, and statistical analysis was performed to compare the age between the delirium positive and negative groups.

\section{Age-matched group analysis}

After the evaluation of incidence in all the patients, each patient with delirium (age-matched delirium positive group) was matched with two patients of the same age in the delirium negative group (age-matched delirium negative group). The selection of patients from the database of spine surgeries was carried out by one of the co-authors who was not informed of the patients' backgrounds. If there was no patient of the same age, we selected patients within 2 years of age difference. Risk factors in the agematched delirium positive group were compared with those in the age-matched delirium negative group. Preoperative risk factors included age, sex, ambulatory status, number of medication, smoking history, habit of alcohol use, history of surgery under general anesthesia, comorbidities (diabetes mellitus, cardiovascular disease, and neurological disorder), white blood cell (WBC) count and C-reactive protein (CRP), $\mathrm{Hb}, \mathrm{Hct}$, Alb, and sodium ( $\mathrm{Na}$ ) levels. Intraoperative factors included cervical surgery, operative time, and intraoperative blood loss. Postoperative factors included WBC count and CRP, $\mathrm{Hb}, \mathrm{Alb}$, and $\mathrm{Na}$ levels on postoperative day 1 and highest body tempera- 
Table 1. Incidence of postoperative delirium overall and according to age in the delirium positive and negative groups Age group (yr) No. of delirium positive group No. of delirium negative group Incidence of postoperative delirium (\%)

\begin{tabular}{lrrr}
$<60$ & 0 & 139 & 0 \\
$60-64$ & 3 & 56 & 5.1 \\
\hline $65-69$ & 3 & 79 & 3.7 \\
\hline $70-74$ & 9 & 83 & 9.8 \\
\hline $75-79$ & 18 & 73 & 39.8 \\
\hline $80-84$ & 21 & 38 & 50.6 \\
\hline $85-89$ & 5 & 5 & 11.1 \\
\hline Overall & 59 & 473 & \\
\hline
\end{tabular}

ture until the delirium or within 7 postoperative days. All the data were obtained from medical or nursing records. No patient data was lost.

\section{Data analysis}

All the data were analyzed using JMP ver. 9.0.3 for Macintosh (SAS Institute Inc., Cary, NC, USA). The mean age of the delirium positive and negative groups is presented as a five-number summary, whereas other numerical variables are presented as the mean and standard deviation. The Mann-Whitney $U$-test was used for the statistical analysis of the difference in the mean values between the groups for non-normally distributed and ordinal variables. The Student $t$-test was employed for normally distributed variables. The chi-square test was used to compare categorical variables. A $p$-value of $<0.05$ was considered statistically significant. Multivariate logistic regression models, with backward elimination, were constructed to find independent risk factors associated with the occurrence (positive or negative) of postoperative delirium. Factors with $p<0.05$ in the previous univariate analysis were included. Odds ratios and their $95 \%$ confidence intervals were calculated. A $p$-value of $<0.05$ was considered statistically significant.

\section{Results}

\section{Incidence and characteristics of delirium in all patients}

Delirium occurred after spine surgery in 59 of 532 patients (11.1\%). The age-specific incidence of delirium and mean age at the onset of delirium (for age of 60-89 years presented in 5-year intervals) were calculated (Table 1). The incidence of delirium increased with increasing age.

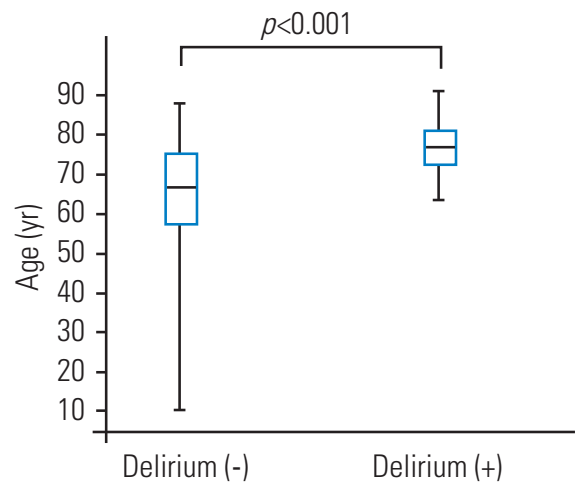

\begin{tabular}{lccccccc} 
& Mean \pm SD & Minimum & $25 \%$ & Median & $75 \%$ & $\begin{array}{c}\text { Maxi- } \\
\text { mum }\end{array}$ \\
\hline Delirium (-) & $62.5 \pm 17.3$ & 10 & 57 & 67 & 74 & 89 \\
\hline Delirium (+) & $77.6 \pm 6.6$ & 62 & 74 & 78 & 82 & 89 \\
\hline
\end{tabular}

Fig. 1. Age distribution and mean age in the delirium negative and positive groups. The upper graph shows the age distribution of each group, presented as a five-number summary. The lower table presents the mean $\pm S D$ age, minimum, first quartile, median, third quartile, and maximum age for each group. A significant difference in age is seen between the two groups $(p<0.001)$. SD, standard deviation.

In the $80-84$ and $85-89$ years age groups, the incidence was $35.6 \%$ and $50.0 \%$, respectively (Table 1 ). The mean age of the delirium positive group was significantly higher than that of the delirium negative group (77.6 \pm 6.6 versus $62.5 \pm 17.3$ years, respectively; $p<0.001$ ) (Fig. 1 ). Almost all the cases of delirium were observed between the day of the surgery and three days after it. Two patients had delirium on postoperative days 4 and 5. All the patients recovered from the delirium by postoperative 1 week. 


\section{Univariate analysis in the age-matched groups}

The age-matched delirium positive group included 59 patients, while the age-matched delirium negative group included 116 patients. Univariate analysis was initially performed. Regarding preoperative data, male sex, cervi-

Table 2. Univariate analysis of patient background and intraoperative data in the age-matched delirium positive and delirium negative groups

\begin{tabular}{|c|c|c|c|}
\hline Factor & $\begin{array}{l}\text { Age-matched delirium positive } \\
\text { group }(n=59)\end{array}$ & $\begin{array}{l}\text { Age-matched delirium } \\
\text { negative group }(n=116)\end{array}$ & $p$-value \\
\hline Mean age (yr) & $77 \pm 6$ & $77 \pm 6$ & \\
\hline Male sex & 36 & 56 & 0.086 \\
\hline Cervical surgery (\%) & $22(37.9)$ & $29(25.0)$ & 0.077 \\
\hline Operative time (min) & $145 \pm 83$ & $121 \pm 72$ & 0.055 \\
\hline Amount of intraoperative bleeding $(\mathrm{mL})$ & $251 \pm 333$ & $113 \pm 168$ & $0.004^{*}$ \\
\hline Inability to ambulate (\%) & $10(17.2)$ & $8(6.9)$ & 0.06 \\
\hline No. of medication types & $7.4 \pm 4.0$ & $7.4 \pm 4.6$ & 0.58 \\
\hline Smoking history (\%) & $3(5.2)$ & $13(11.2)$ & 0.17 \\
\hline Habit of alcohol use (\%) & $17(29.3)$ & $27(23.3)$ & 0.39 \\
\hline History of surgery (\%) & $42(72.4)$ & $81(69.8)$ & 0.72 \\
\hline Diabetes mellitus (\%) & $8(13.8)$ & $18(15.5)$ & 0.76 \\
\hline Cardiovascular disease (\%) & $13(22.4)$ & $25(21.6)$ & 0.90 \\
\hline Brain disease $(\%)$ & $9(15.5)$ & $10(8.6)$ & 0.18 \\
\hline
\end{tabular}

Values are presented as mean \pm standard deviation or number (\%).

* $p<0.05$ (statistically significant difference).

Table 3. Univariate analysis of preoperative and postoperative laboratory data and highest postoperative body temperatures in the age-matched delirium positive and negative groups

\begin{tabular}{|c|c|c|c|}
\hline Factor & $\begin{array}{c}\text { Age-matched delirium } \\
\text { positive group }\end{array}$ & $\begin{array}{l}\text { Age-matched delirium } \\
\text { negative group }\end{array}$ & $p$-value \\
\hline \multicolumn{4}{|l|}{ Preoperative } \\
\hline WBC (cells/mL) & $6,055 \pm 1,948$ & $5,996 \pm 3,622$ & 0.90 \\
\hline $\mathrm{CRP}(\mathrm{mg} / \mathrm{dL})$ & $0.50 \pm 0.95$ & $0.32 \pm 0.86$ & 0.22 \\
\hline $\mathrm{Hb}(\mathrm{g} / \mathrm{dL})$ & $12.7 \pm 1.60$ & $13.0 \pm 1.48$ & 0.32 \\
\hline $\operatorname{Hct}(\%)$ & $37.9 \pm 4.50$ & $38.6 \pm 4.13$ & 0.29 \\
\hline $\mathrm{Na}(\mathrm{mmol} / \mathrm{L})$ & $139.3 \pm 5.5$ & $140.6 \pm 2.9$ & $0.04^{*}$ \\
\hline Alb (g/dL) & $3.8 \pm 0.6$ & $3.9 \pm 0.4$ & 0.25 \\
\hline \multicolumn{4}{|l|}{ Postoperative } \\
\hline WBC (cells/mL) & $8,115 \pm 3,186$ & $8,500 \pm 2,550$ & 0.39 \\
\hline $\mathrm{CRP}(\mathrm{mg} / \mathrm{dL})$ & $3.12 \pm 3.83$ & $1.80 \pm 1.73$ & $0.002^{*}$ \\
\hline $\mathrm{Hb}(\mathrm{g} / \mathrm{dL})$ & $11.1 \pm 1.87$ & $11.8 \pm 3.47$ & 0.14 \\
\hline $\operatorname{Hct}(\%)$ & $32.5 \pm 5.4$ & $34.5 \pm 4.8$ & $0.02^{*}$ \\
\hline $\mathrm{Na}(\mathrm{mmol} / \mathrm{L})$ & $136.7 \pm 4.8$ & $137.7 \pm 3.4$ & 0.11 \\
\hline Alb $(g / d L)$ & $3.1 \pm 0.5$ & $3.3 \pm 0.4$ & $0.004^{*}$ \\
\hline Body temperature (degrees Celsius) & $37.6 \pm 0.5$ & $37.4 \pm 0.4$ & $0.009^{*}$ \\
\hline
\end{tabular}

Values are presented as the mean \pm standard deviation.

WBC, white blood cells; CRP, C-reactive protein; Hb, hemoglobin; Hct, hematocrit; Na, sodium; Alb, albumin. ${ }^{*} p<0.05$ (statistically significant difference). 
cal operation, and nonambulatory status tended to be associated with delirium $(p<0.1)$, but the association was not statistically significant. There was no significant difference in the number of medications, history of smoking and alcohol use, history of surgery under general anesthesia, and comorbidities (diabetes mellitus, cardiovascular disease, and neurological disease) (Table 2). Regarding the preoperative laboratory data, blood $\mathrm{Na}$ levels were significantly lower in the age-matched delirium positive group than in the age-matched delirium negative group $(p=0.04)$. Other factors, including WBC count and CRP, Hb, Hct, and Alb levels, were not significant (Table 3). Regarding intraoperative data, blood loss was significantly higher in the age-matched delirium positive group than in the agematched delirium negative group $(p=0.004)$. Longer operative times tended to be associated with delirium $(p=0.055)$ (Table 2). Regarding postoperative data, high CRP levels, low Hct and Alb levels, and high body temperature on postoperative day 1 were identified as significant risk factors for delirium $(p=0.002,0.02,0.004$, and 0.009 , respectively) (Table 3).

\section{Multivariate analysis in the age-matched groups}

Subsequently, multivariate logistic regression analysis was performed. Preoperative Na levels; intraoperative blood loss; postoperative CRP, Hct, and Alb levels; and body temperature (detected as significant risk factors of delirium in the univariate analysis) were analyzed. Intraoperative blood loss was the only significant risk factor identified (odds ratio, 7.07; 95\% confidence interval, 0.02-0.97) (Table 4).

\section{Discussion}

Common symptoms of postoperative delirium are disarray, hallucination, and delusion. Patients may become hyperactive or hypoactive. Adverse outcomes have been reported in association with postoperative delirium. $\mathrm{Pa}$ tients and their families are negatively affected during hospitalization, and the condition may lead to increased mortality rates, accidents (e.g., falls and self-removal of intravenous lines), impediment to treatment, attrition of medical staff, higher medical care expenses, and elongation of hospital stay [9-12]. Moreover, after discharge, patients who experience postoperative delirium have a high likelihood of presenting to a nursing facility due to further onset of other complications or recurrence of delirium $[13,14]$. Thus, postoperative delirium leads to worse treatment outcomes. However, the mechanisms and prevention methods for postoperative delirium remain unclear.

Many studies have reported on postoperative delirium. Dasgupta et al. [15] reported an incidence rate of $47 \%$ after cardiac surgery and 5.1\%-52\% after noncardiac surgery. In orthopedics, the incidence rate of postoperative delirium has been reported to be $28 \%-61 \%$ after general orthopedic surgery, 26\%-61\% after hip fracture surgery, and $13 \%-41 \%$ after total hip arthroplasty [16]. The incidence seems to vary according to the type of surgery. After spine surgery, the incidence of postoperative delirium has been reported to be $3.3 \%-18 \%$ [17-19]. The variation in incidence may be attributed to the differences in operative stress, such as the duration of surgery and anesthesia, blood loss, pain severity, and duration of bed rest postoperatively. Therefore, research aimed at the detection of risk factors for postoperative delirium should focus on the operation type. We only analyzed patients who underwent spine surgery. In our study, the incidence of postopera-

Table 4. Multivariate analysis of significant risk factors on univariate analysis of age-matched delirium positive and negative groups

\begin{tabular}{lcc} 
Factor & Odds ratio $(95 \%$ confidence interval) & $p$-value \\
\hline Intraoperative amount of bleeding & $0.14(0.02-0.97)$ & $0.046^{*}$ \\
\hline Preoperative low sodium level & $13.4(0.43-519.02)$ & 0.142 \\
\hline Postoperative high serum C-reactive protein level & $0.72(0.01-33.84)$ & 0.870 \\
Postoperative low hematocrit level & $1.08(0.1-8.58)$ & 0.944 \\
\hline Postoperative low albumin level & $2.66(0.22-33.27)$ & 0.439 \\
\hline Postoperative high body temperature & $0.25(0.02-2.50)$ & 0.236 \\
\hline
\end{tabular}

* $p<0.05$ (statistically significant difference). 
tive delirium was $11.1 \%, 15.0 \%$, and $21.0 \%$ in all patients, those aged $>60$ years, and those aged $>70$ years, respectively. These rates were relatively similar to those reported in recent previous studies on postoperative delirium after spine surgery [17,20,21].

Numerous risk factors have been reported to be related to postoperative delirium. Almost all studies have suggested that older age is a risk factor for postoperative delirium; some studies have targeted patients aged $>65$ or 70 years at the beginning of the study. In the present study, older age was associated with a higher incidence of postoperative delirium. Based on these findings, older age can be considered one of the strongest risk factors for postoperative delirium. We performed an age-matched control study to eliminate the influence of age on other risk factors.

In the current study, severe bleeding was found to be a significant intraoperative risk factor for postoperative delirium ( $p=0.004)$, but longer operative time was not $(p=0.055)$. In a meta-analysis of risk factors for postoperative delirium after spine surgery [6], both severe bleeding and longer operative time were found to be risk factors for postoperative delirium. Generally, longer operative time is associated with large amounts of bleeding, and the two factors are possibly confounding. Severe bleeding induces anemia, which decreases oxygen transport to the brain, resulting in declined brain function. The findings of our study indicated that to prevent delirium after spinal surgery, efforts should be taken to reduce intraoperative bleeding as much as possible, especially in elderly patients.

A limitation of this study was that we did not examine some risk factors that have been previously reported, such as the types of medication used preoperatively and degree of pain.

\section{Conclusions}

In the present study, we performed an age-matched analysis to identify the risk factors for postoperative delirium after spine surgery for eliminating the influence of age as a risk factor. Large amounts of intraoperative bleeding, low preoperative serum Na levels, high postoperative serum CRP levels, low Hct and Alb levels, and high body temperature were found to be significant risk factors in the univariate analysis. Large amounts of intraoperative bleeding remained a risk factor for postoperative delirium in the multivariate analysis. We should be aware of and take precautions against the occurrence of postoperative delirium after spine surgery in patients of older age and experiencing severe intraoperative bleeding.

\section{Conflict of Interest}

No potential conflict of interest relevant to this article was reported.

\section{Author Contributions}

Tadao Morino: conception and design; Masayuki Hino: data acquisition and analysis; Shintaro Yamaoka, Hiroshi Misaki, Hiroshi Imai: data acquisition; Tadanori Ogata: critical revision; and Hiromasa Miura: supervision.

\section{References}

1. American Psychiatric Association. Diagnostic and statistical manual of mental disorders. 5th ed. Washington (DC): American Psychiatric Association; 2013.

2. Brown C 4th, Deiner S. Perioperative cognitive protection. Br J Anaesth 2016;117(suppl 3):iii52-61.

3. Koster S, Hensens AG, Schuurmans MJ, van der Palen J. Risk factors of delirium after cardiac surgery: a systematic review. Eur J Cardiovasc Nurs 2011;10:197-204.

4. Oh ES, Li M, Fafowora TM, et al. Preoperative risk factors for postoperative delirium following hip fracture repair: a systematic review. Int J Geriatr Psychiatry 2015;30:900-10.

5. Bin Abd Razak HR, Yung WY. Postoperative delirium in patients undergoing total joint arthroplasty: a systematic review. J Arthroplasty 2015;30:1414-7.

6. Shi C, Yang C, Gao R, Yuan W. Risk factors for delirium after spinal surgery: a meta-analysis. World Neurosurg 2015;84:1466-72.

7. American Psychiatric Association. Diagnostic and statistical manual of mental disorders: text revision (DSM-IV-TR). Washington (DC): American Psychiatric Association; 1994.

8. Whitlock EL, Vannucci A, Avidan MS. Postoperative delirium. Minerva Anestesiol 2011;77:448-56.

9. Inouye SK, Bogardus ST Jr, Charpentier PA, et al. A multicomponent intervention to prevent delirium in hospitalized older patients. N Engl J Med 
1999;340:669-76.

10. Lawlor PG, Gagnon B, Mancini IL, et al. Occurrence, causes, and outcome of delirium in patients with advanced cancer: a prospective study. Arch Intern Med 2000;160:786-94.

11. Litaker D, Locala J, Franco K, Bronson DL, Tannous Z. Preoperative risk factors for postoperative delirium. Gen Hosp Psychiatry 2001;23:84-9.

12. Fong TG, Tulebaev SR, Inouye SK. Delirium in elderly adults: diagnosis, prevention and treatment. Nat Rev Neurol 2009;5:210-20.

13. Schuurmans MJ, Duursma SA, Shortridge-Baggett LM. Early recognition of delirium: review of the literature. J Clin Nurs 2001;10:721-9.

14. Inouye SK, Westendorp RG, Saczynski JS. Delirium in elderly people. Lancet 2014;383:911-22.

15. Dasgupta M, Dumbrell AC. Preoperative risk assessment for delirium after noncardiac surgery: a systematic review. J Am Geriatr Soc 2006;54:1578-89.

16. O'Keeffe ST, Ni Chonchubhair A. Postoperative delirium in the elderly. Br J Anaesth 1994;73:673-87.
17. Fineberg SJ, Nandyala SV, Marquez-Lara A, Oglesby M, Patel AA, Singh K. Incidence and risk factors for postoperative delirium after lumbar spine surgery. Spine (Phila Pa 1976) 2013;38:1790-6.

18. Kawaguchi $Y$, Kanamori M, Ishihara H, et al. Postoperative delirium in spine surgery. Spine J 2006;6:1649.

19. Ushida T, Yokoyama T, Kishida Y, et al. Incidence and risk factors of postoperative delirium in cervical spine surgery. Spine (Phila Pa 1976) 2009;34:2500-4.

20. Jiang X, Chen D, Lou Y, Li Z. Risk factors for postoperative delirium after spine surgery in middle- and old-aged patients. Aging Clin Exp Res 2017;29:103944.

21. Soh S, Shim JK, Song JW, Kim KN, Noh HY, Kwak YL. Postoperative delirium in elderly patients undergoing major spinal surgery: role of cerebral oximetry. J Neurosurg Anesthesiol 2017;29:426-32. 